\title{
A Survival Case of Fulminant Right-Side Dominant Eosinophilic Myocarditis
}

\author{
Keishi Moriwaki, ${ }^{1}$ MD, Kaoru Dohi, ${ }^{1}$ MD, Taku Omori, ${ }^{1}$ MD, \\ Muneyoshi Tanimura, ${ }^{1}$ MD, Emiyo Sugiura, ${ }^{1}$ MD, Shiro NaKamori,,${ }^{1}$ MD, \\ Toshiki SAWAI, ${ }^{1,2}$ MD, Kyoko ImanaKa-Yoshida, ${ }^{3,4}$ MD, \\ Norikazu YAmADA, ${ }^{1} \mathrm{MD}$, and Masaaki Ito, ${ }^{1} \mathrm{MD}$
}

\begin{abstract}
SUMMARY
A 59-year-old Japanese woman was admitted to a nearby hospital with dyspnea and general malaise. Transthoracic echocardiography revealed right ventricular (RV) dilatation with severely reduced systolic function and leftward shift of the intraventricular septum. She was initially diagnosed with acute right heart failure, and fell into cardiogenic shock requiring an intra-aortic balloon pump and inotropic agents. An endomyocardial biopsy (EMB) demonstrated extensive interstitial edema, infiltration of inflammatory cells including numerous eosinophils, and myocytolysis with eosinophil degranulation. She was histologically diagnosed with eosinophilic myocarditis. Steroid pulse therapy was initiated, and her hemodynamic status improved along with dramatic recovery of the RV function. EMB 6 days after the initiation of steroid pulse therapy showed the disappearance of infiltration and degranulation of eosinophils, although lymphocytic infiltration still remained. Positron emission tomography-computed tomography (PET/CT) 23 days after steroid pulse therapy showed an increased ${ }^{18} \mathrm{~F}$-FDG uptake in the intraventricular septum and left ventricle, suggesting persistent myocardial inflammation. She was then treated with a maintenance dose of prednisolone. She became free of symptoms and follow-up echocardiography showed normal cardiac function 3 months after the initiation of corticosteroid treatment. In addition, EMB and PET/CT showed no inflammation. This is the first case report of fulminant and right-sided dominant eosinophilic myocarditis successfully treated with corticosteroid. (Int Heart J 2017; 58: 459-462)
\end{abstract}

Key Words: Right ventricular dysfunction, Eosinophil degranulation, Corticosteroid therapy

$\mathrm{M}$ yocarditis generally impairs both ventricles equally, and predominant right-sided heart failure is very rare. ${ }^{1)}$ Since it was reported that the loss of right ventricular (RV) function is the most powerful predictor of death or cardiac transplantation in myocarditis, ${ }^{2)}$ early diagnosis increases the chances for effective treatment. Corticosteroid therapy is recommended as the treatment for eosinophilic myocarditis, although there is limited consensus regarding the use, dose, and duration of corticosteroid therapy. Here, we report a case of fulminant right-side dominant eosinophilic myocarditis in a 59-year-old Japanese woman who was successfully treated with corticosteroid therapy.

\section{Case Report}

A 59-year-old Japanese woman visited a nearby hospital with chest discomfort. She was previously healthy, had no medical or surgical history, took no medications, and had no known drug allergies. A 12-lead electrocardiogram (ECG) showed premature ventricular contraction without ST eleva- tion, and bisoprolol was prescribed. However, she was admitted to the same hospital with dyspnea and general malaise 10 days after the first visit. Her blood pressure was $92 / 49 \mathrm{mmHg}$, pulse rate was $90 \mathrm{bpm}$, and her temperature was $36.9^{\circ} \mathrm{C}$. Laboratory tests showed a normal leukocyte count $(7600 / \mu \mathrm{L}$; normal value, $3400-8700 / \mu \mathrm{L}$ ) with a normal absolute eosinophil count, and elevated levels of C-reactive protein $(0.77 \mathrm{mg} / \mathrm{dL}$; normal value, $0-0.3 \mathrm{mg} / \mathrm{dL})$, troponin I (1.62 ng/mL; normal value, $0-0.04)$, and B-type natriuretic peptide $(806 \mathrm{pg} / \mathrm{mL}$; normal value, 0-18.4 pg/mL). ECG showed ST elevation in leads II, III, aVF, and V1 to V5 (Figure 1A). A chest X-ray showed cardiomegaly and pulmonary congestion. Transthoracic echocardiography revealed RV dilatation with severely reduced systolic function and leftward shift of the intraventricular septum. The left ventricular (LV) systolic function was also mildly reduced with an LV ejection fraction (LVEF) of about $50 \%$. Right heart catheterization (RHC) revealed elevated right atrial pressure (RAP) and pulmonary capillary wedge pressure (PCWP) to the same extent (mean RAP: $14 \mathrm{mmHg}$ and mean PCWP: $15 \mathrm{mmHg}$ ), and the cardiac index was $2.2 \mathrm{~L} /$ minute/ $\mathrm{m}^{2}$. Although the LV pulse pressure was preserved, that in the

From the ${ }^{1}$ Department of Cardiology and Nephrology, Mie University Graduate School of Medicine, ${ }^{2}$ Department of Cardiology, Nagai Hospital, ${ }^{3}$ Department of Pathology and Matrix Biology, Mie University Graduate School of Medicine, and ${ }^{4}$ Mie University Research Center for Matrix Biology, Tsu, Japan.

Address for correspondence: Kaoru Dohi, MD, Department of Cardiology and Nephrology, Mie University Graduate School of Medicine 2-174 Edobashi, Tsu, Mie 514-8507, Japan. E-mail: dohik@ clin.medic.mie-u.ac.jp

Received for publication July 21, 2016. Revised and accepted September 26, 2016.

Released in advance online on J-STAGE May 12, 2017.

All rights reserved by the International Heart Journal Association. 

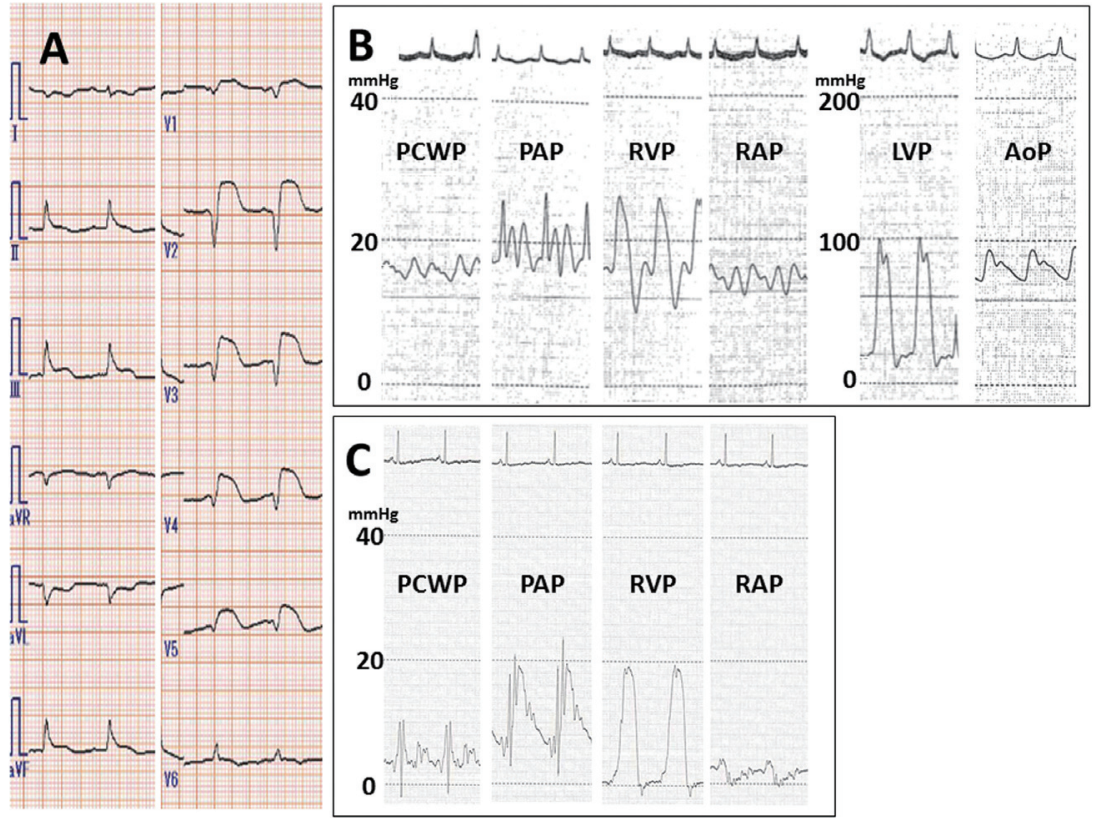

Figure 1. A 12-lead ECG showed ST elevation in leads II, III, aVF, and V1 to V5 (A). Right heart catheterization revealed elevated right atrial pressure (RAP) and pulmonary capillary wedge pressure (PCWP), and reduced pulse pressure in the right ventricle (B). RAP, PCWP, and right ventricular pulse pressure normalized 3 months after the initiation of corticosteroid treatment (C). PCWP indicates pulmonary capillary wedge pressure; PAP, pulmonary arterial pressure; RVP, right ventricular pressure; RAP, right atrial pressure; LVP, left ventricular pressure; and AoP, arterial pressure.
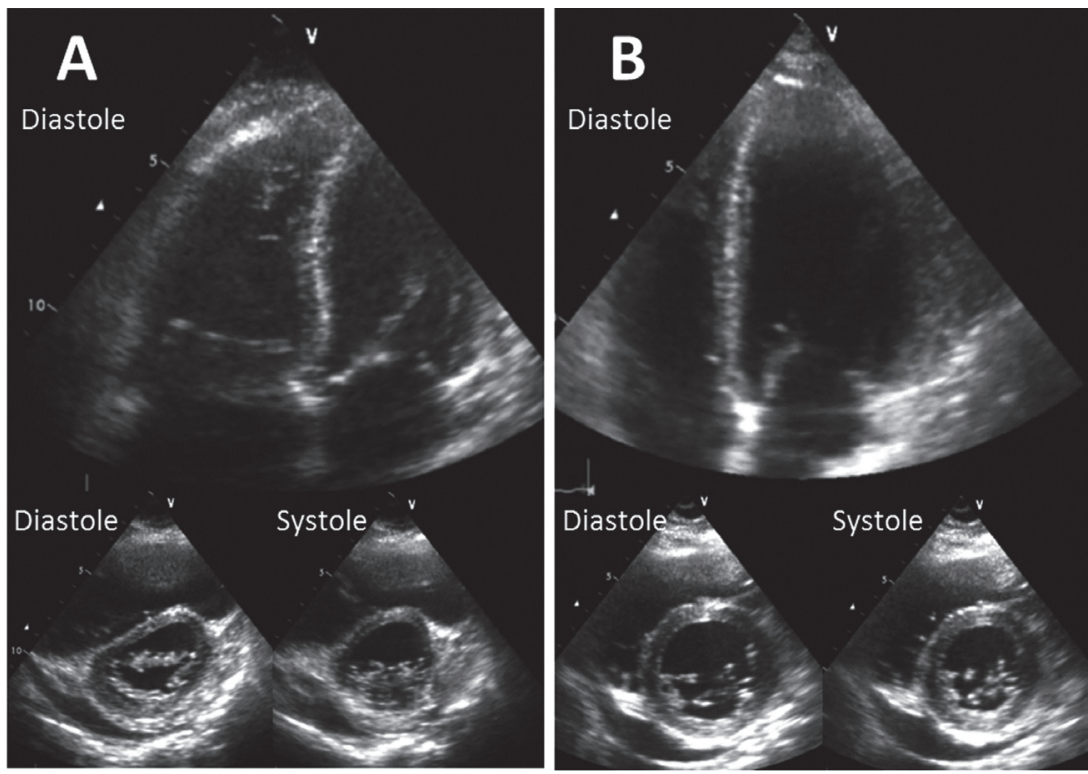

Figure 2. Transthoracic echocardiography in the apical 4-chamber view and parasternal short-axis view before (A) and 9 days after (B) the initiation of steroid pulse therapy. The right ventricle was dilated and markedly hypokinetic, with a marked leftward shift of the intraventricular septum (A). Right ventricular function recovered dramatically while left ventricular ejection fraction remained unchanged (B).

right ventricle was reduced (Figure 1B). Coronary angiography showed normal coronary arteries and an endomyocardial biopsy (EMB) was performed.

She was initially diagnosed with acute right heart failure of unknown etiology, and inotropic agents including dobutamine and dopamine were administered. She fell into cardiogenic shock and required the insertion of an intra-aortic balloon pump on the next day of hospital admission. The patient's hemodynamic condition did not improve, and she was transferred to our hospital for intensive therapy. RV function assessed by transthoracic echocardiography deteriorated further (Figure 2A).

EMB, which was performed on admission, demonstrated extensive interstitial edema, infiltration of inflammatory cells including numerous eosinophils, and myocytolysis with eosinophil degranulation (Figures 3A and B). She was histologi- 


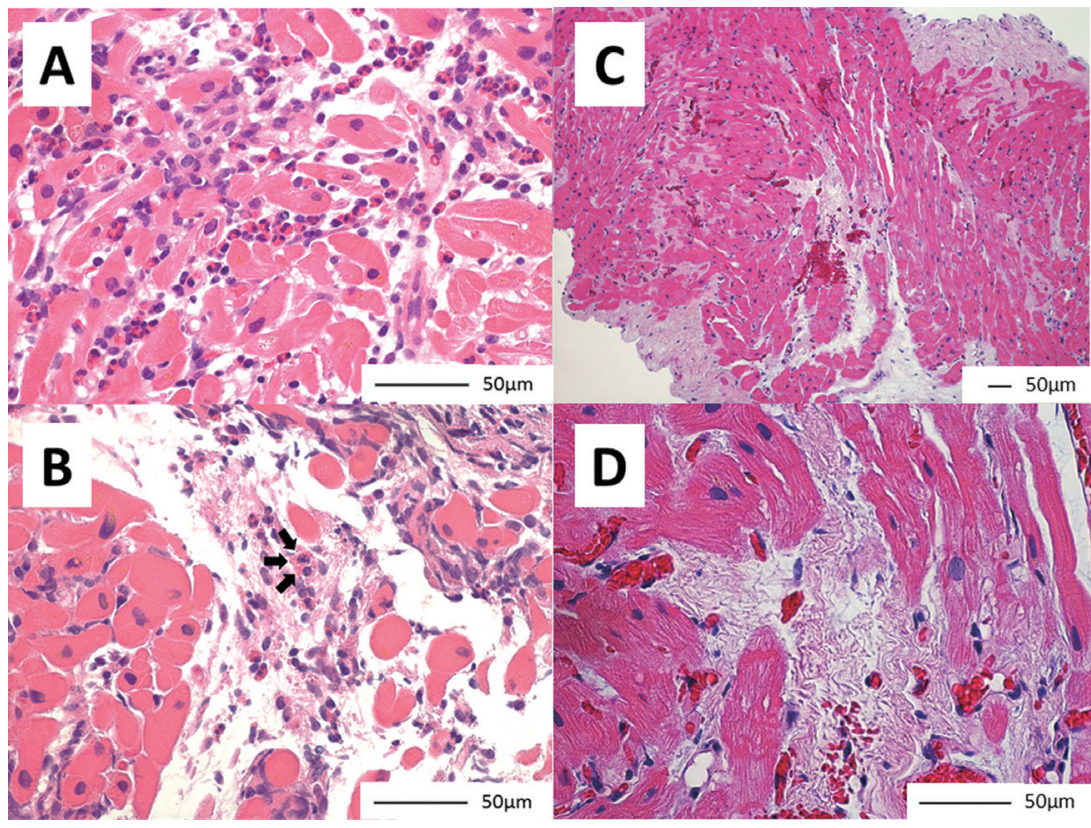

Figure 3. Endomyocardial biopsy (EMB) upon hospital admission showed infiltration of inflammatory cells including numerous eosinophils (A) and eosinophil degranulation (B: arrows). EMB 3 months after the initiation of corticosteroid treatment showed small replacement fibrosis but no inflammatory cells $(\mathbf{C}$ and $\mathbf{D})$.
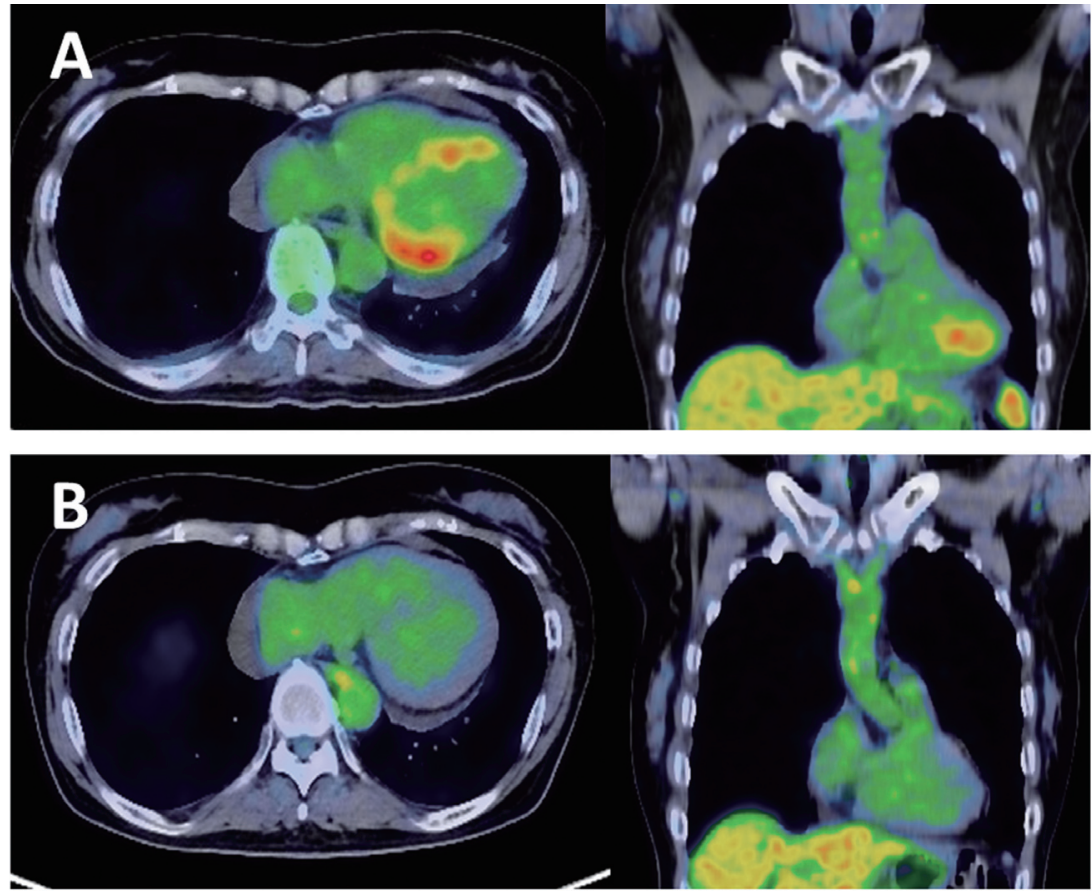

Figure 4. Positron emission tomography-computed tomography (PET/CT) 23 days after the initiation of steroid pulse therapy showed increased ${ }^{18}$ F-FDG uptake in the intraventricular septum and left ventricle (A). ${ }^{18}$ F-FDG uptake disappeared after 3-months of corticosteroid treatment $(\mathbf{B})$.

cally diagnosed with eosinophilic myocarditis. Steroid pulse therapy (methylprednisolone, $1 \mathrm{~g} /$ day IV for 3 days) was initiated, and hemodynamic status improved along with dramatic recovery of the RV function within 9 days (Figure $2 \mathrm{~B}$ ) while LVEF remained unchanged. EMB 6 days after steroid pulse therapy showed disappearance of infiltration and degranulation of eosinophils, although lymphocytic infiltration still remained. Positron emission tomography-computed tomography (PET/ CT) 23 days after steroid pulse therapy showed increased ${ }^{18} \mathrm{~F}$ FDG uptake in the intraventricular septum and left ventricle (Figure 4A). She was then treated with a maintenance dose of prednisolone $(0.5 \mathrm{mg} / \mathrm{kg} / \mathrm{day})$ with gradual tapering to $5 \mathrm{mg} /$ 
day over 3 months. She became free of symptoms, and followup echocardiography and RHC 3 months after the initiation of corticosteroid treatment showed normal cardiac function and normal intra-cardiac pressures (Figure 1C). PET/CT showed no abnormal ${ }^{18} \mathrm{~F}$-FDG uptake (Figure 4B) and EMB revealed small replacement fibrosis but no inflammatory cells (Figures $3 \mathrm{C}$ and D).

Serology testing during admission revealed no signs, symptoms, or objective evidence of specific causes of eosinophilic myocarditis such as parasitic infection, drug hypersensitivity, or vasculitis associated anti-neutrophil cytoplasmic antibody. However, there were increases in anti-Coxsackie B4 and Herpes simplex viral titers. On the other hand, she was also diagnosed with Sjögren's syndrome, which was confirmed by positive results for SS-A/Ro antibodies, Schirmer's test, and lip biopsies.

\section{Discussion}

This is the first case report of fulminant and right-sided dominant eosinophilic myocarditis successfully treated with corticosteroid. The present case showed RV dilatation with severely reduced systolic function and leftward shift of the intraventricular septum on echocardiography. In addition, right heart catheterization revealed elevated right atrial pressure and reduced RV pulse pressure, which were consistent with rightsided heart failure. The patient also had elevated PCWP with pulmonary congestion at presentation. In the setting of acute right heart failure, the heart becomes constrained by the inelastic pericardium secondary to rapid increases in RV and right atrial volume, and which can produce approximate equalization of right atrial, pulmonary venous, and ventricular diastolic pressures. Although the present case did not undergo invasive intra-pericardial pressure measurement, it can be helpful to distinguish pericardial constraint from other causes of elevated PCWP and pulmonary congestion. In the present case, the LV failure may partially contribute to the PCWP elevation because LVEF mildly decreased from the onset of heart failure and FDG-PET/CT showed abnormal ${ }^{18}$ F-FDG uptake in the intraventricular septum and left ventricle even after steroid pulse therapy. The patient was successfully diagnosed with eosinophilic myocarditis by initial EMB, which showed eosi- nophilic degranulation. Although there is limited consensus regarding the use, dose, and duration of corticosteroid therapy in eosinophilic myocarditis, ${ }^{3,4)}$ the refractory RV dysfunction dramatically recovered after corticosteroid therapy. In addition, FDG-PET/CT 23 days after the initiation of steroid pulse therapy showed no uptake of ${ }^{18} \mathrm{~F}-\mathrm{FDG}$ in the RV free wall, suggesting marked regression of myocardial inflammation in the right ventricle. The etiology of the eosinophilic myocarditis remained unidentified in the present case. Although the patient was diagnosed with Sjögren's syndrome during hospitalization, autoimmune myocarditis is rarely complicated with this disease, and the incidence of eosinophilic myocarditis has never been reported. In addition, increases in two elevated viral titers were found, which made it impossible to distinguish between viral and non-viral etiologies.

\section{Disclosures}

K.D. received lecture fees equal to or more than 500,000 yen from Otsuka Pharma Inc. in 2015. N.Y. received lecture fees equal to or more than 500,000 yen from Daiichi Sankyo Co., Ltd. in 2015. M.I. received lecture fees of more than 500,000 yen from Daiichi Sankyo Co., Ltd. and Mochida Pharmaceutical Co., Ltd. in 2015. M.I. received a single-year research grant for the Department of Cardiology and Nephrology, Mie University Graduate School of Medicine, equal to or more than 2 million yen, from Bristol-Myer K.K., Astellas Pharma Inc. and BIOTRONIK Japan, Inc. in 2015.

No relevant conflicts of interest related to the article were disclosed by the authors.

\section{REFERENCES}

1. Mancio J, Bettencourt N, Oliveira M, Pires-Morais G, Ribeiro VG. Acute right ventricular myocarditis presenting with chest pain and syncope. BMJ Case Rep 2013; 2013: bcr2012007173.

2. Cooper LT Jr. Myocarditis. N Engl J Med 2009; 360: 1526-38.

3. Magnani JW, Dec GW. Myocarditis: current trends in diagnosis and treatment. Circulation 2006; 113: 876-90. (Review)

4. Yanagisawa T, Inomata T, Watanabe I, et al. Clinical significance of corticosteroid therapy for eosinophilic myocarditis. Int Heart J 2011; 52: 110-3. 